\title{
Y-27632, a ROCK Inhibitor, Improved Laser-induced Shock Wave (LISW)-induced Cochlear Synaptopathy in Mice
}

\section{Yutaka Koizumi}

Yamagata University Faculty of Medicine: Yamagata Daigaku Igakubu Daigakuin Igakukei Kenkyuka Kunio Mizutari ( $\nabla$ tari@mbf.ocn.ne.jp )

National Defense Medical College: Boei lka Daigakko https://orcid.org/0000-0002-0340-8293

\section{Satoko Kawauchi}

National Defense Medical College: Boei Ika Daigakko

\section{Shunichi Sato}

National Defense Medical College: Boei Ika Daigakko

\section{Akihiro Shiotani}

National Defense Medical College: Boei Ika Daigakko

\section{Seiji Kakehata}

Yamagata University Faculty of Medicine: Yamagata Daigaku Igakubu Daigakuin Igakukei Kenkyuka

\section{Research}

Keywords: Cochlea, Rho-associated coiled-coil containing protein kinase (ROCK), synapse, regeneration, Y-27632, hearing loss, inner ear

Posted Date: April 13th, 2021

DOl: https://doi.org/10.21203/rs.3.rs-404746/v1

License: (9) This work is licensed under a Creative Commons Attribution 4.0 International License. Read Full License 


\section{Abstract}

Recently, a pathological condition called cochlear synaptopathy has been clarified, and as a disorder of the auditory nerve synapses that occurs prior to failure of hair cells it has been recognized as a major cause of sensorineural hearing loss. However, cochlear synaptopathy is untreatable. Inhibition of rhoassociated coiled-coil containing protein kinase (ROCK), a serine-threonine protein kinase, has been reported to have neuroprotective and regenerative effects on synaptic pathways in the nervous system, including those in the inner ear. We previously demonstrated the regenerative effect of the ROCK inhibitor, Y-27632, on an excitotoxic cochlear nerve damage model in vitro. In this study, we aimed to validate the effect of ROCK inhibition on mice with cochlear synaptopathy induced by laser-induced shock wave (LISW) in vivo. After the elevation of ROCK1/2 expression in the damaged cochlea was confirmed, we administered Y-27632 locally via the middle ear. The amplitude of wave I in the auditory brainstem response and the number of synapses in the Y-27632-treated cochlea increased significantly. These results clearly demonstrate that ROCK inhibition has a promising clinical application in the treatment of cochlear synaptopathy, which is the major pathology of sensorineural hearing loss.

\section{Introduction}

Primary cochlear neural degeneration, which is also called cochlear synaptopathy, is recognized as a common pathology associated with sensorineural hearing loss [1], which is caused by various etiologies such as noise [2], aging [3], congenital genetic factors [4], and blast exposure [5]. A unique characteristic of this pathology is that it is not accompanied by the loss of hair cells, which are the primary receptors of sound. The clinical characteristic of this pathology is also unique in that it causes mild or sometimes no hearing threshold shift, but it is closely related to the pathogenesis of tinnitus [6, 7] and hyperacusis [8]. Cochlear synaptopathy, popularly called as "hidden hearing loss," has now been recognized as the critical therapeutic target, because quite a few proportions of patients with hearing impairment suffer from this pathology, and it is still untreatable $[9,10]$.

Rho-associated coiled-coil containing protein kinase (ROCK), a serine-threonine protein kinase, is a target protein of small molecular weight GTP-binding protein Ras homolog (Rho) [11], and activation of the ROCK pathway has been associated with inhibition of neurite regeneration and outgrowth in patients with spinal cord injury [12]. In contrast, inhibition of the Rho/ROCK pathway has an effect on axonal regeneration in the peripheral nerves, including the cochlear nerve $[13,14]$. In our previous study, we examined the effects of ROCK inhibitors on the damaged auditory nerve end and synapses using an excitotoxic cochlear organotypic model and found that ROCK inhibitors could regenerate the cochlear nerve axons and synapses between the inner hair cell $(\mathrm{IHC})$ and the auditory nerve after excitotoxic injury of the cochlea [15]. Based on these findings, we hypothesized that ROCK inhibitors would exert their effects on synaptic remodeling even in an in vivo model of cochlear synaptopathy.

To validate this hypothesis, we examined the effects of ROCK inhibitors on the damaged synapse between IHC and the auditory nerve using a cochlear synaptopathy model generated by a laser-induced 
shock wave (LISW). The LISW-induced hearing dysfunction model was developed as a blast-induced hearing loss model that replicates a blast-exposed ear with pure sensorineural hearing loss without any conductive hearing loss [5]. The biggest advantage of this animal model is that LISW can reduce the number of synapses without any hair cell loss, which is the most characteristic pathology of cochlear synaptopathy, with good reproducibility. We examined the changes in ROCK expression in the cochlea with synaptopathy and then administered a ROCK inhibitor to the damaged cochlea locally. Herein, we report the therapeutic effect of a ROCK inhibitor on cochlear synaptopathy in vivo.

\section{Results}

\section{ROCK1 and ROCK2 expression in the organ of Corti after LISW-induced cochlear damage}

In this study, we used a mouse model of cochlear synaptopathy induced by direct LISW exposure of the inner ear. The output level of LISW was set to induce cochlear synaptopathy, which is associated with synaptic degeneration without hair cell loss, according to a previous study [5], and we then reproduced synaptic loss in mice without both inner and outer hair cell loss.

First, we examined the expression of ROCK1 and ROCK2 in the cochlea before and 1 day after LISW exposure. In the native cochlea, ROCK1 was expressed around the outer hair cell area; however, no expression was observed around the IHC area (Fig. 1a, a', a"). After LISW exposure, ROCK1 expression was obviously elevated in both the inner and outer hair cell areas (Fig. 1b, b'). Around the IHC area, elevated ROCK1 expression was observed ubiquitously around the end of the NF200-positive peripheral axons (Fig. 1b', b', around the purple area). Additionally, elevation of ROCK1 expression was observed in the cytoplasm of the outer hair cells (Fig. 1b). The expression pattern of ROCK2 in the native cochlea was similar to that of ROCK1, and weak expression was observed in the outer hair cell area. After cochlear damage, ROCK2 expression was increased at the end of the NF200-positive peripheral axon area (Fig. 1d, $d^{\prime}, d^{\prime \prime}$ ). The characteristic punctiform elevation pattern of ROCK2 was observed (Fig. 1d, white arrowheads).

We subsequently confirmed the changes in mRNA expression of ROCK1, ROCK2, and RhoA, which is upstream effector of ROCKs in the Rho/ROCK pathway a day after cochlear damage. Quantitative polymerase chain reaction (PCR) revealed that the mRNA expression levels of RhoA, ROCK1, and ROCK2 increased significantly after LISW exposure (4.923-fold, $p=0.013 ; 3.235$-fold, $p=0.031$; and 5.656-fold, $p$ $=0.045$, respectively, two-tailed Mann-Whitney U test) (Fig. 1e).

\section{Effect of Y-27632 on the degenerated synapse induced by LISW exposure}

After we confirmed the upregulation of the Rho/ROCK pathway induced by cochlear damage, we used the ROCK inhibitor, Y-27632, to rescue cochlear synaptopathy after LISW exposure. First, we measured the mRNA expression of RhoA, ROCK1, and ROCK2 two days after the local administration of $10 \mathrm{mM} \mathrm{Y}$ 27632 to the cochlea. All of them were significantly more suppressed by local Y-27632 treatment than by 
sham surgery (RhoA: 0.4480 folds, $p=0.0064$; ROCK1: $0.4716, p=0.0463$; ROCK2: 0.5995 folds, $p=$ 0.0486, two-tailed Mann-Whitney U test; Fig. 2b).

Next, we measured synaptic markers in the inner hair cells, including presynaptic ribbons stained by CtBP2 and postsynaptic puncta labeled by GluA2 to explore the functional connection between the IHC and auditory peripheral axons 1 month after local treatment with Y-27632. The numbers of CtBP2 puncta and GluA2 puncta in the sham surgery group were significantly lower than those in the control group (without LISW exposure) at all frequencies, except at $5.6 \mathrm{kHz}$ (CtBP2: two-way ANOVA, $\mathrm{F}_{5,120}=4.344, p=$ 0.0011 ; Bonferroni multiple comparison significance at $8.0 \mathrm{kHz}[p=0.005]$, at $11.3 \mathrm{kHz}[p<0.001]$, at 16.0 $\mathrm{kHz}[p<0.001]$, at $22.65 \mathrm{kHz}$ [ $p<0.001]$, at $32.00 \mathrm{kHz}$ [ $p<0.001]$; Fig. 2f) (GluA2: two-way ANOVA, $\mathrm{F}_{5,120}=$ $3.373, p=0.007$; Bonferroni multiple comparison significance at $8.0 \mathrm{kHz}[p<0.001]$, at $11.3 \mathrm{kHz}[p<$ $0.001]$, at $16.0 \mathrm{kHz}$ [ $p<0.001]$, at $22.6 \mathrm{kHz}$ [ $p<0.001]$, at $32.0 \mathrm{kHz}[p<0.001]$; Fig. $2 \mathrm{~g}$ ). Enlarged images focusing on the synapses in the inner hair cell area (Fig. 2c, c') showed a decrease in the number of presynaptic ribbons (CtBP2-immunoreactive puncta, red) and also confirmed the presence of orphan synapses (Fig. 2c, C', red arrows), which is the synaptic structure lacking apposed glutamate-receptor patches (GluA2 immunoreactive puncta, green). In the $1 \mathrm{mM}$ Y-27632-treated group, the numbers of CtBP2 puncta and GluA2 puncta were still lower at any frequency than those in the sham surgery group; however, the trend of increase in both puncta was observed (CtBP2: Bonferroni multiple comparison significance at $5.6 \mathrm{kHz}[p>0.999], 8.0 \mathrm{kHz}[p>0.999]$, at $11.3 \mathrm{kHz}[p>0.999]$, at $16.0 \mathrm{kHz}[p=0.752]$, at $22.6 \mathrm{kHz}[p=0.719]$, at $32.0 \mathrm{kHz}[p>0.999]$; Fig. 2d, d', f) (GluA2: Bonferroni multiple comparison significance at $5.6 \mathrm{kHz}[p>0.999], 8.0 \mathrm{kHz}[p>0.999]$, at $11.3 \mathrm{kHz}[p>0.999]$, at $16.0 \mathrm{kHz}[p=0.592]$, at $22.6 \mathrm{kHz}[p=0.207]$, at $32.0 \mathrm{kHz}[p>0.168]$; Fig. $2 \mathrm{~d}, \mathrm{~d}$ ', g). Otherwise, the numbers of CtBP2 puncta and

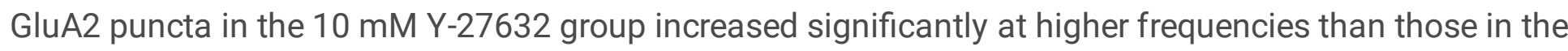
sham surgery group (CtBP2: Bonferroni multiple comparison significance at $16.0 \mathrm{kHz}$ [ $p=0.003]$, at 22.6 $\mathrm{kHz}[p<0.001]$, at $32.0 \mathrm{kHz}$ [ $p=0.001]$; Fig. 2e, e', f) (GluA2: Bonferroni multiple comparison significance at $16.0 \mathrm{kHz}[p<0.001]$, at $22.6 \mathrm{kHz}[p<0.001]$, at $32.0 \mathrm{kHz}$ [ $p<0.001]$; Fig. 2e, e', g). These results suggest that Y-27632 has a dose-dependent effect on synaptic regeneration in LISW-induced cochlear synaptopathy.

\section{Effect of Y-27632 on the sensorineural hearing dysfunction after LISW exposure}

Finally, we conducted the cochlear function test, which includes auditory brainstem responses (ABRs) and distortion product otoacoustic emissions (DPOAEs), before and after exposure to LISW and the ROCK inhibitor, Y-27632, at several time points (Fig. 3). In the sham surgery group, the ABR and DPOAE thresholds were temporarily elevated immediately after LISW exposure; however, the threshold was recovered 7 days after sham surgery with no significant difference at all frequencies tested (Fig. 3a, g). Although the elevation of $A B R$ threshold was recovered, the ABR wave I amplitude decreased from 1 day after LISW exposure and continued to decrease up to 28 days after sham surgery with significant differences from $8.00 \mathrm{kHz}$ to $22.65 \mathrm{kHz}$ (two-way ANOVA, $\mathrm{F}_{5,120}=46.20, p=0.007$; Bonferroni multiple comparison significance at $8.00 \mathrm{kHz}[p<0.001]$, at $11.33 \mathrm{kHz}[p<0.001]$, at $16.00 \mathrm{kHz}[p<0.001]$, at 22.65 $\mathrm{kHz}[p<0.014] ;$ Fig. 3d). These results clearly showed that LISW exposure in this research setting could 
induce pure cochlear synaptopathy, which is the pathology of ABR wave I amplitude elevation without ABR/DPOAE threshold elevation.

Similar to the sham surgery group, the $1 \mathrm{mM}$ Y-27632-treated group did not show a permanent ABR/DPOAE threshold shift (Fig. 3b, h). In addition, the ABR wave I amplitude at 28 days after $1 \mathrm{mM} \mathrm{Y-}$ 27632 treatment continued to decrease and was significantly different from that before LISW exposure (two-way ANOVA, $F_{5,120}=23.13, p<0.001$ ); however, this parameter at $22.65 \mathrm{kHz}$ was not significantly different from that before LISW exposure at the same frequency (Bonferroni multiple comparison significance at $22.65 \mathrm{kHz}$ [ $p=0.916]$; Fig. 3e). Interestingly, the ABR wave I amplitude 28 days after exposure to $10 \mathrm{mM}$ Y-27632 was not significantly different from that before LISW exposure at all frequencies tested (two-way ANOVA, $F_{5,120}=23.13$, Bonferroni multiple comparison significance at all frequencies [ $p>0.999]$; Fig. 3f), as well as the ABR/DPOAE threshold (ABR: two-way ANOVA, $\mathrm{F}_{5,120}=$ 15.08, Bonferroni multiple comparison significance at all frequencies [ $p>0.999]$; Fig. 3c) (DPOAE: twoway ANOVA, $F_{5,120}=22.68$, Bonferroni multiple comparison significance at all frequencies [ $p>0.999$ ]; Fig. 3i). These results also suggest that the ROCK inhibitor, Y-27632, has a dose-dependent effect on the recovery of hearing function in cochlear synaptopathy.

\section{Discussion}

In this study, we showed the involvement of the Rho/ROCK pathway in the synapse degeneration and recovery effect of a ROCK inhibitor on cochlear synaptopathy induced by LISW exposure. We observed an increase in mRNA expression levels of RhoA, ROCK1, and ROCK2 in the cochlea immediately after LISW exposure and localized expression of ROCKs in the organ of Corti. Although the increase in mRNA expression levels was transient, the changes in mRNA expression levels and localized expression of ROCKs were similar to the results of our previous in vitro study that used the excitotoxic cochlear damage model [15]. We also found that the local administration of the ROCK inhibitor, Y-27632, to the inner ear could promote synaptic reorganization and recover hearing function, as confirmed by a re-increase in ABR wave I amplitude. In this study, we adopted local administration of ROCK inhibitor onto the round window membrane via surgery to deliver the drug into the cochlea. Drug delivery via the middle ear is an established method both in animal studies [16] and in clinical settings [17]. This is the first report to prove that a ROCK inhibitor could recover synapses between IHC and the auditory nerve and restore hearing function in an in vivo model of cochlear synaptopathy.

In this study, we used the LISW mouse model to induce the pathology of cochlear synaptopathy; however, conventionally, acute cochlear synaptopathy is induced by noise exposure in animal models. By using noise-induced cochlear synaptopathy, the damaged area, where synapses decrease, is generally limited in the cochlear basal turn $[2,18]$. The biggest advantage of the LISW-induced cochlear synaptopathy model is that the synapse decreased area without hair cell loss is wider toward the lower frequency area [5]. As shown in Figs. 2 and 3, our model showed synaptic loss and ABR wave I amplitude decrease in the entire cochlear area. Owing to this characteristic, we analyzed the effect of treatment in the cochlear area in detail. In addition, as shown in Fig. 1, the pattern of ROCK expression in the organ of Corti in the LISW 
model was similar to that in the excitotoxic synapse and neuron damage model in vitro, which showed that elevated ROCK1 expression was observed ubiquitously around the end of peripheral axons, and ROCK2 expression was increased at the end of the axon area as a punctiform pattern [15]. Cochlear excitotoxicity has been hypothesized to represent a critical aspect of cochlear synaptopathy [19]. Therefore, our mouse model is an ideal platform for developing new treatment strategies for cochlear synaptopathy.

Since the pathology of cochlear synaptopathy has been clarified in recent years, there have been several reports on treatment based on this pathology, that is, protection or regeneration of IHC-auditory nerve synapses. Neurotrophic factors, such as brain-derived neurotrophic factor (BDNF) [20], neurotrophin-3 (NT-3) [21, 22], and growth factors (insulin-like growth factor 1 (IGF1) [23]. Among these, neurotrophic factors have been studied for a relatively long time and have been shown to be effective for the treatment of hearing loss [24], but they are difficult to use because they show insufficient permeability at the bloodbrain and blood-nerve barriers [25]. The tropomyosin receptor kinase (Trk) receptor is a high-affinity receptor for neurotrophic factors. TrkA binds to nerve growth factor (NGF) [26], BDNF, and neurotrophin4/5 (NT-4/5) [27, 28], and TrkC binds to NT-3 [29]. Small-molecule TrkB receptor agonists have the ability to restore noise-induced hidden hearing loss, similar to its ligand, BDNF [30]. ROCK inhibitors are involved in a cascade of neurotrophic factors. The ROCK pathway is downstream of these neurotrophins [31, 32]. Therefore, ROCK inhibition may be a direct mechanism for synapse regeneration in cochlear synaptopathy. In addition, most candidate compounds have not been proven to be safe for use in clinical settings. Therefore, it would be easier to apply a ROCK inhibitor for neuronal regeneration in the clinical setting because Y-27632 is already in clinical use. Our results demonstrate a possible breakthrough therapeutic strategy for hearing impairment accompanied by primary synaptic degeneration.

\section{Methods}

\section{Animals}

Forty-five CBA/J mice (male, 6 weeks old) weighing 17-20 g were purchased from the Japan Charles River (Yokohama, Japan). The animals were provided free access to water and were fed a regular diet. Moreover, they were individually housed and maintained at $23-25^{\circ} \mathrm{C}$. In this study, all procedures on mice were performed under general anesthesia induced by intramuscular injection of ketamine (75 mg/kg) and medetomidine ( $1 \mathrm{mg} / \mathrm{kg}$ ). All experimental procedures reported herein were approved by the Institutional Animal Care and Use Committee of the National Defense Medical College and were performed in accordance with the guidelines of the National Institutes of Health and the Ministry of Education, Culture, Sports, Science and Technology of Japan (approval \#18050). All efforts were made to minimize the number of animals used and their suffering.

\section{LISW irradiation of the inner ear}

LISWs were generated as described previously [5]: a laser target was irradiated with a 532-nm Q-switched neodymium-doped yttrium aluminum garnet (Nd: YAG) laser (Brilliant b, Quintal, Les Ulis Cerdex, France; 
pulse width, 6 nanoseconds). Specifically, the laser target was a 10-mm in diameter, $0.5-\mathrm{mm}$ thick black natural rubber disk, and a 1.0-mm thick transparent polyethylene terephthalate sheet was bonded to the top of the target area to confine the laser-induced plasma, by which the LISW impulse was increased. The laser pulse was focused to a 4.0-mm diameter spot on the laser target using a plano-convex lens. The laser fluence on the target was set at $2.0 \mathrm{~J} / \mathrm{cm}^{2}$, as described in a previous report [5], to generate the cochlear synaptopathy model without hair cell loss. Temporal pressure waveforms of LISWs were measured with a hydrophone (HNR-1000, Onda Co., Sunnyvale, CA, USA). The signals of the hydrophone were recorded using a digital oscilloscope (DP04104B, Tektronix, Tokyo, Japan; bandwidth, $1 \mathrm{GHz}$ ), and they were calibrated using software provided by the manufacturer of the pressure sensor.

\section{Administration of Y-27632, a ROCK inhibitor, to the inner ear}

Y-27632 (257-00511, Wako Pure Chemical Industries Ltd, Osaka, Japan), was dissolved in water, and the concentration was adjusted to $1 \mathrm{mM}$ and $10 \mathrm{mM}$. PBS was used as the sham control. The following three groups were compared to investigate the effects of the ROCK inhibitor, Y-27632, on the LISW-induced cochlear neuropathic model: 1 . sham surgery group (treated with PBS); 2. ROCK inhibition group (1 mM Y27632); and 3. ROCK inhibition group (10 mM Y-27632). To administer Y-27632 to the inner ear, the left postauricular region of the mice was positioned under a stereomicroscope. After a 20-mm postauricular skin incision was made, subcutaneous tissues and superficial fascia were dissected, and the otic bulla was exposed. Tympanotomy was performed using microforceps, and the hole was enlarged to allow clear observation of the round window niche. Then, $1 \mu \mathrm{L}$ of PBS or ROCK inhibitor was injected into the round window niche using a Nanoject III Programmable Nanoliter injector (3-000-207, Drummond Scientific Company, Broomall, PA, USA).

\section{Cochlear function tests}

Cochlear function tests were performed in each animal at six log-spaced frequencies (half-octave steps from 5.6 to $32.0 \mathrm{kHz}$ ) before and 1 day, 7 days, and 28 days after blast exposure. Mice were anesthetized with ketamine ( $75 \mathrm{mg} / \mathrm{kg}$ i.p.) and medetomidine hydrochloride ( $1 \mathrm{mg} / \mathrm{kg}$ i.p.). For ABRs, needle electrodes were inserted at the vertex and pinna, with the ground near the tail. ABRs were evoked with 5$\mathrm{ms}$ tone pips (0.5-ms rise-fall with a cos2 onset envelope delivered at 35/s). The response was amplified, filtered, and averaged using a LabVIEW-driven data-acquisition system. The sound level was raised in $5 \mathrm{~dB}$ steps from $\geq 10 \mathrm{~dB}$ to $<80 \mathrm{~dB}$ SPL. At each sound level, 1024 responses were averaged (with alternating stimulus polarity). On visual inspection of stacked waveforms, the "ABR threshold" was defined as the lowest SPL level at which any wave could be detected, which usually corresponds to the level step just below that at which the peak-to-peak response amplitude rose significantly above the noise floor. When no response was observed at the highest sound level available, the threshold was designated as $5 \mathrm{~dB}$ greater than that level so that statistical tests could be performed. For amplitude versus level functions, the wave I peak was identified by visual inspection at each sound level, and the peak-to-peak amplitude was computed. 
For the measurement of DPOAEs at $2 f 1-f 2$, the primary tones were set such that the frequency ratio $(\mathrm{f} 2 / \mathrm{f} 1)$ was 1.2 and the $\mathrm{f} 2$ level was $10 \mathrm{~dB}$ below the $\mathrm{f} 1$ level. For each $\mathrm{f} 2 / \mathrm{f} 1$ primary pair, levels were swept in $5 \mathrm{~dB}$ steps from 20 to $80 \mathrm{~dB}$ SPL (for f2). At each level, both waveform and spectral averaging were used to increase the signal-to-noise ratio of the recorded ear-canal sound pressure, and the amplitude of the DPOAE at $2 \mathrm{f} 1-\mathrm{f} 2$ was extracted from the averaged spectra, along with the noise floor at nearby points in the spectrum. Iso-response curves were interpolated from the plots of the DPOAE amplitude versus sound level. The threshold was defined as the $\mathrm{f} 1$ level required to produce a DPOAE at 0 dB SPL.

\section{Quantitative RT-PCR}

Harvested cochlear tissues were collected and stored until further analysis (Ambion, Austin, TX, USA). Total RNA was extracted using the RNeasy Mini Kit (Qiagen, Valencia, CA, USA) according to the manufacturer's instructions. Quantitative RT-PCR was performed on a Thermal Cycler Dice Real Time System using the One Step SYBR PrimeScript PLUS RT-PCR Kit (RR096A; TaKaRa Bio, Shiga, Japan). Forward $(F)$ and reverse $(R)$ primer sequences were RhoA-F, 5'-AGCTTGTGGTAAGACATGCTTG-3' and RhoA-R, 5'-GTGTCCCATAAAGCCAACTCTAC-3'; ROCK1-F, 5'-GACTGGGGACAGTTTTGAGAC-3' and ROCK1R, 5'-GGGCATCCAATCCATCCATCCAGC-3'; and ROCK2-F, 5'-TTGGTTCGTCATAAGGCATCAC-3' and ROCK2R, 5'-TGTTGGCAAAGGCCATAATATCT-3'. PCR cycling conditions included 40 cycles of $95^{\circ} \mathrm{C}$ for $5 \mathrm{~s}$ and $60^{\circ} \mathrm{C}$ for $30 \mathrm{~s}$. Relative mRNA expression levels were determined using the $\triangle \Delta \mathrm{Ct}$ method with glyceraldehyde 3-phosphate dehydrogenase (GAPDH) as an internal control. All reactions were performed in duplicate. For assessment, relative mRNA expression levels were standardized to cochlear samples extracted before LISW.

\section{Cochlear processing and immunohistochemistry}

Mice were perfused transcardially with $0.5 \mathrm{~mL} / \mathrm{g}$ of lactated Ringer's solution followed by $1 \mathrm{~mL} / \mathrm{g}$ of $4 \%$ paraformaldehyde (PFA)/0.1 M phosphate buffer (PB) at room temperature. After decapitation, the cochlea was dissected out, and small holes were made at the round window, oval window, and apex of the cochlea; it was then bathed in $4 \% \mathrm{PFA} / \mathrm{PB}$ at $4{ }^{\circ} \mathrm{C}$ overnight. After decalcification with $0.5 \mathrm{M}$ ethylenediaminetetraacetic acid (EDTA) (Decalcifying Soln. B (EDTA method); Wako Pure Chemical Industries Ltd, Osaka, Japan) for 4 days at $4{ }^{\circ} \mathrm{C}$ with shaking, each cochlea was microdissected into four pieces for whole mount preparation. For immunostaining, cochlear pieces were blocked and permeabilized with $5 \%$ normal horse serum in PBS and $0.3 \%$ Triton $\mathrm{X}-100$ for $1 \mathrm{~h}$ at room temperature, followed by overnight incubation at $37^{\circ} \mathrm{C}$ with the following primary antibodies diluted in $1 \%$ normal horse serum with $0.3 \%$ Triton X-100. Chicken anti-NF 200 (1:500, Millipore, Bedford, MA, USA: AB5539) was used as an auditory nerve marker, rabbit anti-Myo7a (1:500, Proteus Biosciences Inc. Ramona, CA, USA: 25-6790) as a hair cell marker, mouse (IgG1) anti-CtBP2 (1: 500 BD Biosciences, San Jose, CA, USA: 612044) as a presynaptic marker, mouse (IgG2a) anti-GluA2 (1: 2000 Millipore, Bedford, MA, USA:

MAB397) as a postsynaptic marker, and rabbit anti-ROCK1 (1:100 abcam, Cambridge, UK: ab134181) and rabbit anti-ROCK2 (1:100 abcam: ab125025). After washing with PBS, the following secondary antibodies 
diluted 500-fold were incubated at room temperature for $2 \mathrm{~h}$ : Alexa Fluor 350-conjugated goat anti-rabbit IgG (Invitrogen, Carlsbad, CA, USA: A21068), Alexa 488-conjugated goat anti-mouse IgG2a ( A21131; Invitrogen), Alexa 488-conjugated goat anti-rabbit IgG (Invitrogen, A11034), Alexa 568-conjugated goat anti-mouse IgG1 (Invitrogen: A21124), Alexa 647-conjugated goat anti-chicken IgY (Invitrogen: A21449), and rhodamine phalloidin (1:500 Invitrogen, Carlsbad, CA, USA: R415). After washing with PBS, samples were mounted with fluorescence mounting media (Dako, Santa Clara, CA, USA: S3023) and observed.

\section{Quantitative analysis of synapses and hair cells}

The fluorescent-labeled organ of Corti was observed using a BZ-X700 fluorescence microscope (Keyence Corporation, Osaka, Japan) with a water-immersion $60 \times$ objective and $3 \times$ digital zoom. Optical sections in the $x$-y plane ( $z$-sections) were recorded at $0.2 \mu \mathrm{m}$ intervals along the z-axis. The resulting confocal image series ( $z$-stack) contained a three-dimensional image of the entire volume of the cochlear tissues. The z-stack was reconstructed (to view a plane perpendicular to the $x$-y plane) using ImageJ (https://imagej.nih.gov/ij/) or PhotoShop CC (Adobe, San Jose, CA, USA).

To count the number of outer hair cells (OHCs) and IHCs, confocal microscopy was performed at 5.6, 8.0, $11.3,16.0,22.6$, and $32.0 \mathrm{kHz}$ while focusing on the presynaptic ribbons in the basolateral portion of IHCs; an oil-immersion $100 \times$ or water-immersion $60 \times$ objective and a $0.2-\mu \mathrm{m}$ z-step were used. For each frequency region, z-stacks were acquired in each cochlea at three adjacent areas, each containing $\sim 10$ IHCs in a row. The number of OHCs and IHCs per $200 \mu \mathrm{m}$ was counted at each point, as described above. The densities of OHCs and IHCs per $200 \mu \mathrm{m}$ were calculated and compared at each site. The numbers of IHC synaptic ribbons (CtBP2-positive puncta) and glutamate-receptor patches (GluA2 puncta) per $200 \mu \mathrm{m}$ were counted at $5.6,8,11.3,16,22.6$, and $32 \mathrm{kHz}$, as described above. The number of synaptic ribbons and glutamate-receptor patches per IHC was calculated and compared at each site. To minimize bias, the counts were performed by three different individuals who were blinded to the experimental groups.

\section{Statistical analysis}

Statistical analyses were conducted using Prism software (version 7.0; GraphPad software, Inc., La Jolla, CA, USA). For analyses of histological and cochlear function results, two-way analysis of variance (ANOVA), followed by Bonferroni correction for multiple comparisons, was used. The two-tailed MannWhitney $U$ test was employed to compare differences in mRNA expression. The statistical power and the sample size were determined before and after data collection using PS: Power and Sample Size Calculation, Ver. 3.1.6 (Department of Biostatistics, Vanderbilt University, Nashville, TN, USA). Statistical significance was set at $p<0.05$. Error bars represent standard deviation of the mean (SEM).

\section{Abbreviations}

LISW: laser-induced shock wave

ROCK: rho-associated coiled-coil containing protein kinase 
IHC: inner hair cell

ABR: auditory brainstem response

DPOAE: distortion product otoacoustic emissions

BDNF: brain-derived neurotrophic factor

NT-3: neurotrophin-3

IGF1: growth factors (insulin-like growth factor 1

Trk: tropomyosin receptor kinase

PFA: paraformaldehyde

PB: phosphate buffer

EDTA: ethylenediaminetetraacetic acid

OHC: outer hair cells

ANOVA: analysis of variance

SEM: standard deviation of the mean

\section{Declarations}

\section{Ethics approval and consent to participate:}

All animal experiments were approved by the Institutional Animal Care and Use Committee of the National Defense Medical College and were performed in accordance with the guidelines of the National Institutes of Health and the Ministry of Education, Culture, Sports, Science and Technology of Japan (approval \#18050).

\section{Consent for publication:}

Not applicable

\section{Availability of data and materials:}

The datasets used and/or analyzed during the current study are available from the corresponding author on reasonable request.

\section{Competing interests:}

The authors declare no competing financial interests. 


\section{Funding:}

This study was supported by funding from the JSPS KAKENHI (Grant-in-Aid for Scientific Research (B) 20H03833, Fund for the Promotion of Joint International Research (Fostering Joint International Research (B) 20KK0204, Grant-in-Aid for Challenging Exploratory Research 17K19713, and Grant-in-Aid for Early-Career Scientists (B) 17K16887), and a Grant for Advanced Defense Medical Research provided by the Japan Ministry of Defense Grant Number A-4.

\section{Author contributions:}

S.Kakehata organized the study. YK, KM, and S.Kakehata were involved in the conceptualization and design of the study. YK and KM conducted the research and analyzed the data. S.Kawauchi, S.S., and A.S. provided material and laboratory support. YK wrote the early drafts of this paper. KM prepared the manuscript. All authors have reviewed the final version of the manuscript.

\section{Acknowledgment:}

We thank Dr. Eiko Kimura for his research assistance.

\section{Conflict of interest:}

The authors have declared that no conflict of interest exists.

\section{References}

1. Kujawa SG, Liberman MC. Synaptopathy in the noise-exposed and aging cochlea: Primary neural degeneration in acquired sensorineural hearing loss. Hear Res. 2015;330:191-9.

2. Kujawa SG, Liberman MC. Adding insult to injury: cochlear nerve degeneration after "temporary" noise-induced hearing loss. J Neurosci. 2009;29:14077-85.

3. Sergeyenko Y, Lall K, Liberman MC, Kujawa SG. Age-related cochlear synaptopathy: an early-onset contributor to auditory functional decline. J Neurosci. 2013;33:13686-94.

4. Dulon D, Papal S, Patni P, Cortese M, Vincent PF, Tertrais M, et al. Clarin-1 gene transfer rescues auditory synaptopathy in model of Usher syndrome. J Clin Invest. 2018;128:3382-401.

5. Niwa K, Mizutari K, Matsui T, Kurioka T, Matsunobu T, Kawauchi S, et al. Pathophysiology of the inner ear after blast injury caused by laser-induced shock wave. Sci Rep. 2016;6:31754.

6. Schaette R, Turtle C, Munro KJ. Reversible induction of phantom auditory sensations through simulated unilateral hearing loss. PLoS One. 2012;7:e35238.

7. Schaette R, McAlpine D. Tinnitus with a normal audiogram: physiological evidence for hidden hearing loss and computational model. J Neurosci. 2011;31:13452-7.

8. Hickox AE, Liberman MC. Is noise-induced cochlear neuropathy key to the generation of hyperacusis or tinnitus? J Neurophysiol. 2014;111:552-64. 
9. Liberman MC, Kujawa SG. Cochlear synaptopathy in acquired sensorineural hearing loss: Manifestations and mechanisms. Hear Res. 2017;349:138-47.

10. Aedo C, Aguilar E. Cochlear synaptopathy: new findings in animal and human research. Rev Neurosci. 2020;31:605-15.

11. Madaule P, Axel R. A novel ras-related gene family. Cell. 1985;41:31-40.

12. Gopalakrishnan SM, Teusch N, Imhof C, Bakker MH, Schurdak M, Burns DJ, et al. Role of Rho kinase pathway in chondroitin sulfate proteoglycan-mediated inhibition of neurite outgrowth in PC12 cells. J Neurosci Res. 2008;86:2214-26.

13. Lie M, Grover M, Whitlon DS. Accelerated neurite growth from spiral ganglion neurons exposed to the Rho kinase inhibitor H-1152. Neuroscience. 2010;169:855-62.

14. Defourny J, Poirrier AL, Lallemend F, Mateo Sanchez S, Neef J, Vanderhaeghen P, et al. EphrinA5/EphA4 signalling controls specific afferent targeting to cochlear hair cells. Nat Commun. 2013;4:1438.

15. Koizumi Y, Ito T, Mizutari K, Kakehata S. Regenerative Effect of a ROCK Inhibitor, Y-27632, on Excitotoxic Trauma in an Organotypic Culture of the Cochlea. Front Cell Neurosci. 2020;14:572434.

16. Mizutari K, Fujioka M, Hosoya M, Bramhall N, Okano HJ, Okano H, et al. Notch inhibition induces cochlear hair cell regeneration and recovery of hearing after acoustic trauma. Neuron. 2013;77:58-69.

17. Rauch SD, Halpin CF, Antonelli PJ, Babu S, Carey JP, Gantz BJ, et al. Oral vs intratympanic corticosteroid therapy for idiopathic sudden sensorineural hearing loss: a randomized trial. JAMA. 2011;305:2071-9.

18. Fernandez KA, Guo D, Micucci S, De Gruttola V, Liberman MC, Kujawa SG. Noise-induced Cochlear Synaptopathy with and Without Sensory Cell Loss. Neuroscience. 2020;427:43-57.

19. Han BR, Lin SC, Espinosa K, Thorne PR, Vlajkovic SM. Inhibition of the Adenosine A2A Receptor Mitigates Excitotoxic Injury in Organotypic Tissue Cultures of the Rat Cochlea. Cells. 2019;8.

20. Sly DJ, Campbell L, Uschakov A, Saief ST, Lam M, O'Leary SJ. Applying Neurotrophins to the Round Window Rescues Auditory Function and Reduces Inner Hair Cell Synaptopathy After Noise-induced Hearing Loss. Otol Neurotol. 2016;37:1223-30.

21. Wang Q, Green SH. Functional role of neurotrophin-3 in synapse regeneration by spiral ganglion neurons on inner hair cells after excitotoxic trauma in vitro. J Neurosci. 2011;31:7938-49.

22. Suzuki J, Corfas G, Liberman MC. Round-window delivery of neurotrophin 3 regenerates cochlear synapses after acoustic overexposure. Sci Rep. 2016;6:24907.

23. Yamahara K, Asaka N, Kita T, Kishimoto I, Matsunaga M, Yamamoto N, et al. Insulin-like growth factor 1 promotes cochlear synapse regeneration after excitotoxic trauma in vitro. Hear Res. 2019;374:5-12.

24. Shinohara T, Bredberg G, Ulfendahl M, Pyykko I, Olivius NP, Kaksonen R, et al. Neurotrophic factor intervention restores auditory function in deafened animals. Proc Natl Acad Sci U S A. 2002;99:165760. 
25. Poduslo JF, Curran GL. Permeability at the blood-brain and blood-nerve barriers of the neurotrophic factors: NGF, CNTF, NT-3, BDNF. Brain Res Mol Brain Res. 1996;36:280-6.

26. Martin-Zanca D, Oskam R, Mitra G, Copeland T, Barbacid M. Molecular and biochemical characterization of the human trk proto-oncogene. Mol Cell Biol. 1989;9:24-33.

27. Ip NY, Stitt TN, Tapley P, Klein R, Glass DJ, Fandl J, et al. Similarities and differences in the way neurotrophins interact with the Trk receptors in neuronal and nonneuronal cells. Neuron. 1993;10:137-49.

28. Klein R, Lamballe F, Bryant S, Barbacid M. The trkB tyrosine protein kinase is a receptor for neurotrophin-4. Neuron. 1992;8:947-56.

29. Lamballe F, Klein R, Barbacid M. trkC, a new member of the trk family of tyrosine protein kinases, is a receptor for neurotrophin-3. Cell. 1991;66:967-79.

30. Fernandez KA, Watabe T, Tong M, Meng X, Tani K, Kujawa SG, et al. Trk agonist drugs rescue noiseinduced hidden hearing loss. JCI Insight. 2021;6.

31. Kaplan DR, Miller FD. Neurotrophin signal transduction in the nervous system. Curr Opin Neurobiol. 2000;10:381-91.

32. Yamashita T, Tucker KL, Barde YA. Neurotrophin binding to the p75 receptor modulates Rho activity and axonal outgrowth. Neuron. 1999;24:585-93.

\section{Figures}




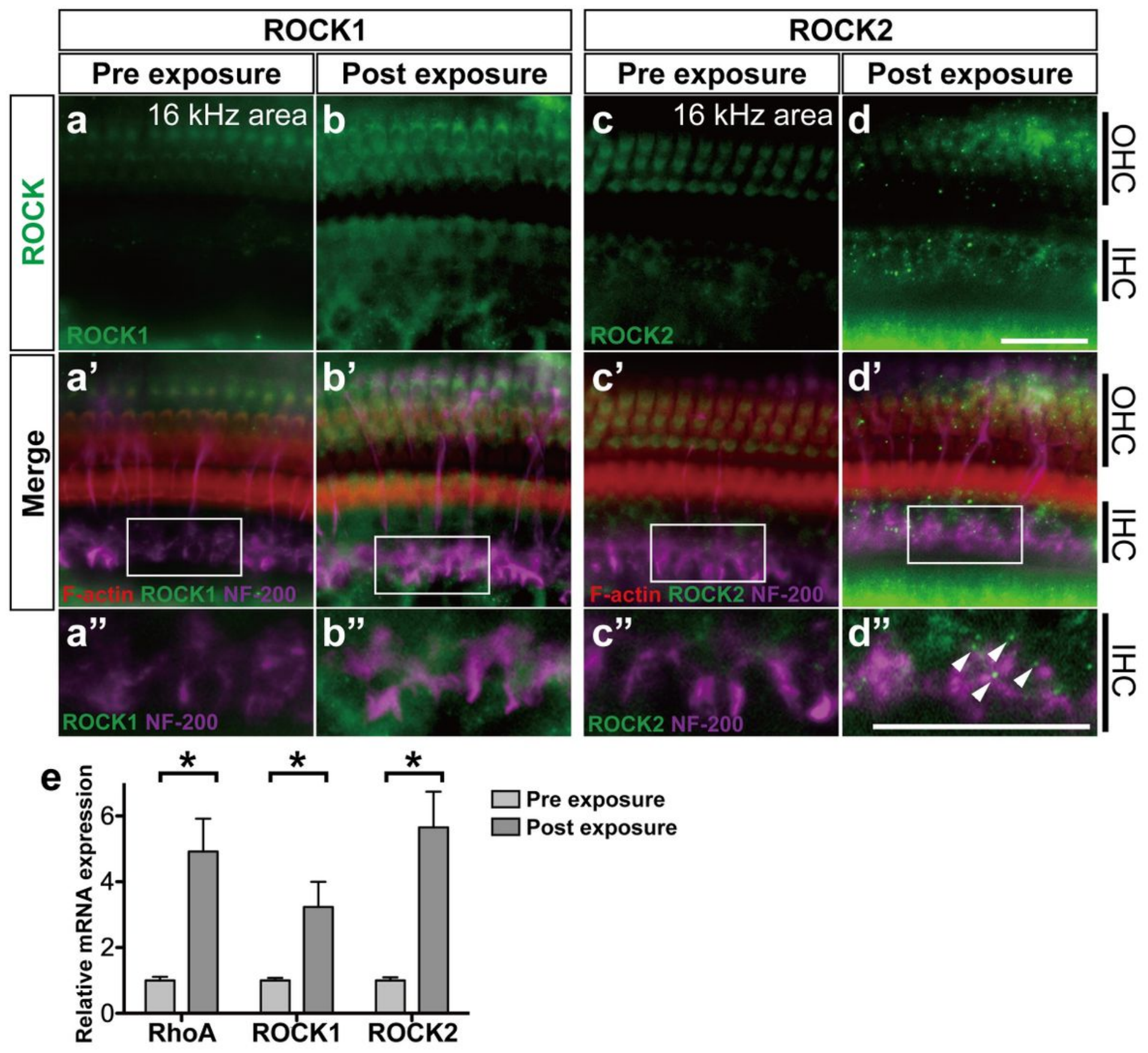

Figure 1

Changes in the expression levels of ROCKs before and after cochlear damage induced by irradiation of the organ of Corti with laser-induced shock wave (LISW). (a-d, a'-d', a"-d") Projection images of a confocal series of immunohistochemistry: tissues of the cochlear organ of Corti form the middle turn (16 kHz area) of the mouse before and 1 day after LISW exposure. Immunohistochemistry for ROCK1 (green, antiROCK1 $(a, b)$, anti-ROCK2 (c, d)) and merged images with F-actin (rhodamine phalloidin, red) and neurofilament (NF-200, purple) are shown in a'-d'. (a"-d") Enlarged images of the white inlets in (a'-d') focusing on the inner hair cells and the end of the peripheral axons of the auditory nerve. After LISW 
exposure, ROCK1 expression showed ubiquitous elevation around the nerve end ( $b$ "), whereas ROCK2 expression showed punctiform enhancement around the inner hair cells (d", white arrowheads) in addition to the ubiquitous elevation of expression. (e) The relative mRNA expression levels of RhoA, ROCK1, and ROCK2 before and 1 day after LISW exposure. The relative mRNA expression levels were standardized by the expression levels before LISW exposure in each mRNA $(n=6)$ * indicates a significant difference $(p<0.05$, two-tailed Mann-Whitney $U$ test $)$. The data are shown as the mean \pm standard errors of mean. IHC; inner hair cell, OHC; outer hair cell. Scale bar: $20 \mu \mathrm{m}$.
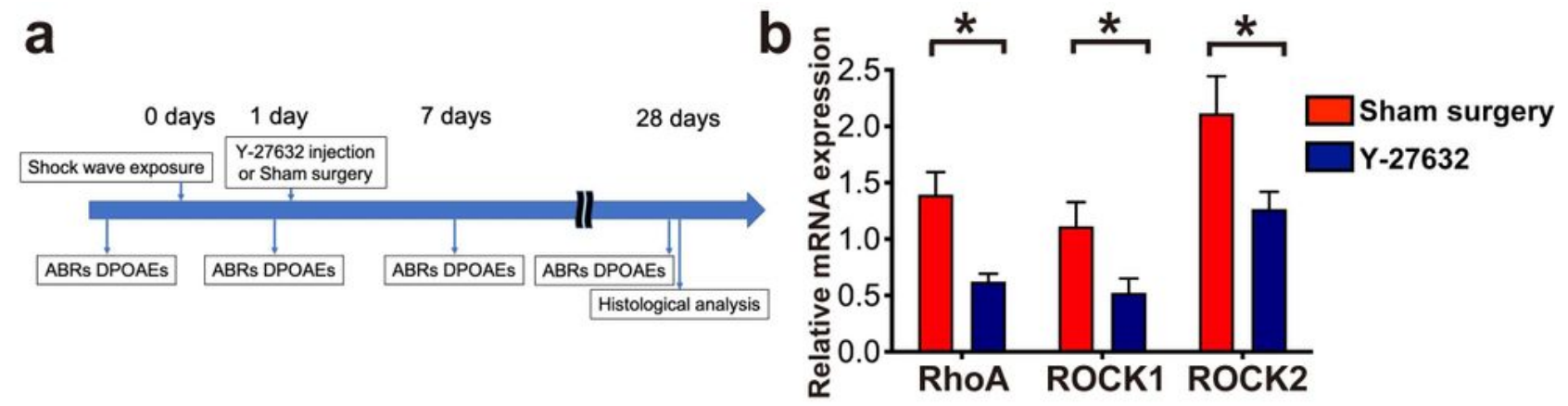

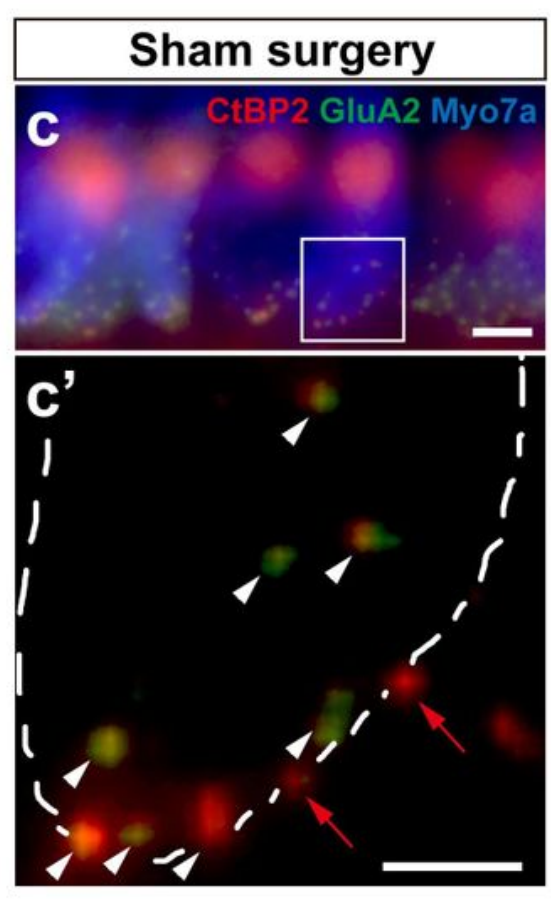

f
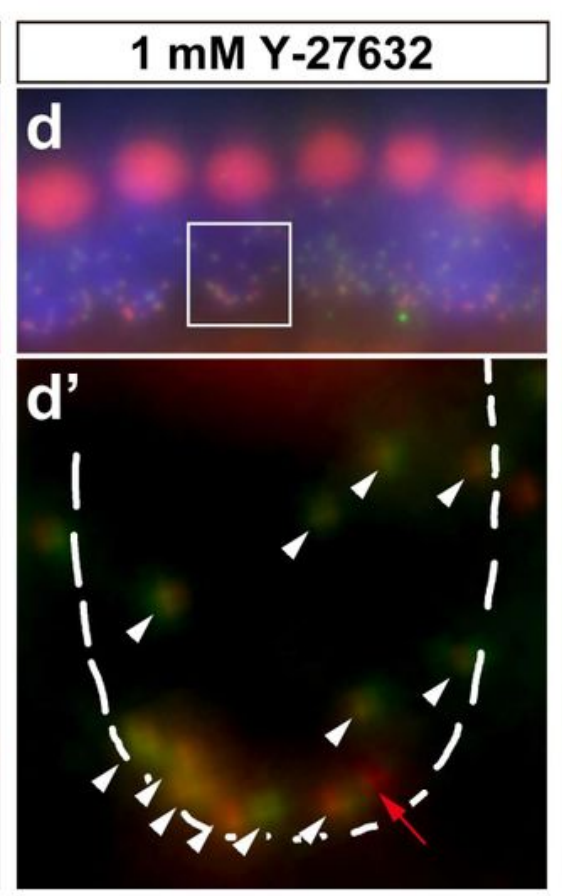

g

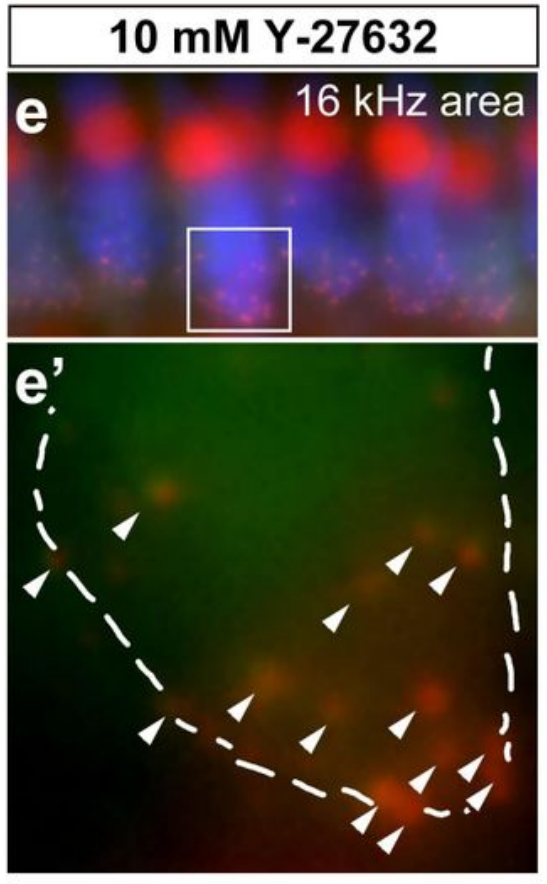

Without LISW exposure Sham surgery 目 $1 \mathrm{mM}$ Y-27632 ㄸm $10 \mathrm{mM}$ Y-27632 * $\stackrel{*}{\mapsto} \stackrel{*}{\mapsto}$

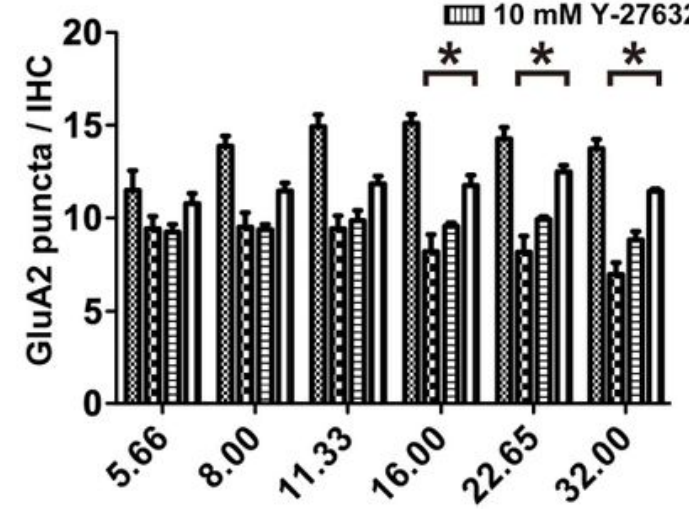

Frequency (kHz) 


\section{Figure 2}

Effect of Y-27632 on the synapses in the inner hair cells after LISW exposure. (a) Timeline of the experiments in this study. (b) The effect of $10 \mathrm{mM} \mathrm{Y-27632} \mathrm{in} \mathrm{the} \mathrm{LISW} \mathrm{exposed} \mathrm{cochleae} \mathrm{on} \mathrm{relative}$ mRNA expression levels of RhoA, ROCK1, and ROCK2, 2 days after Y-27632 treatment. The relative mRNA expression levels were standardized by the expression levels before LISW exposure in each mRNA $(n=6)$. * indicates a significant difference ( $p<0.05$, two-tailed Mann-Whitney $U$ test). (c-e) The effect of $Y-27632$ on the degenerated synapses of the inner hair cells at cochlear $16 \mathrm{kHz}$ area 28 days after treatment with various concentration of Y-27632 (c: without Y-27632, d: $1 \mathrm{mM}$, and e: $10 \mathrm{mM}$ ). In c-e, presynaptic ribbons (CTBP2-immunoreactive puncta, red), postsynaptic densities (GluA2 immunoreactive puncta, green), and hair cells labeled with myosin 7a (blue) are shown. (c'-e') Enlarged images of the white inlets in (c-e) focusing on the synapses in the inner hair cells area. White dotted lines show the contour of the inner hair cells. White arrowhead indicates normal synapse formation with CtBP2-positive patch (red) accompanied with glutamate-receptor patch (green). Red arrow indicates orphan ribbons, which a CtBP2-positive patch with lacking apposed glutamate-receptor patches. In panel c', there are seven normal synapses (white arrow heads) and two orphan synapses (red arrows); in d', there are 12 normal synapses and one orphan synapse; and in e', all 14 synapses have both CtBP2 and glutamate-receptor patch. (f, g) The quantification of the synaptic ribbons (f) and the glutamate receptor $(\mathrm{g})$ observed in the 28 days after treatment and control from single IHC. The number of synapse components is lower in the sham surgery groups than in the control group at all frequencies tested. The number of synapse components is significantly larger in the $10 \mathrm{mM} \mathrm{Y-27632-treated} \mathrm{group} \mathrm{at} \mathrm{higher} \mathrm{frequencies} \mathrm{(asterisks)} \mathrm{than} \mathrm{these} \mathrm{in} \mathrm{the}$ sham surgery group. Scale bar is $5 \mu \mathrm{m}$. * indicates significant differences $(p<0.05$, two-way ANOVA, followed by Bonferroni correction for multiple comparisons). Values are represented as mean \pm SEM. 


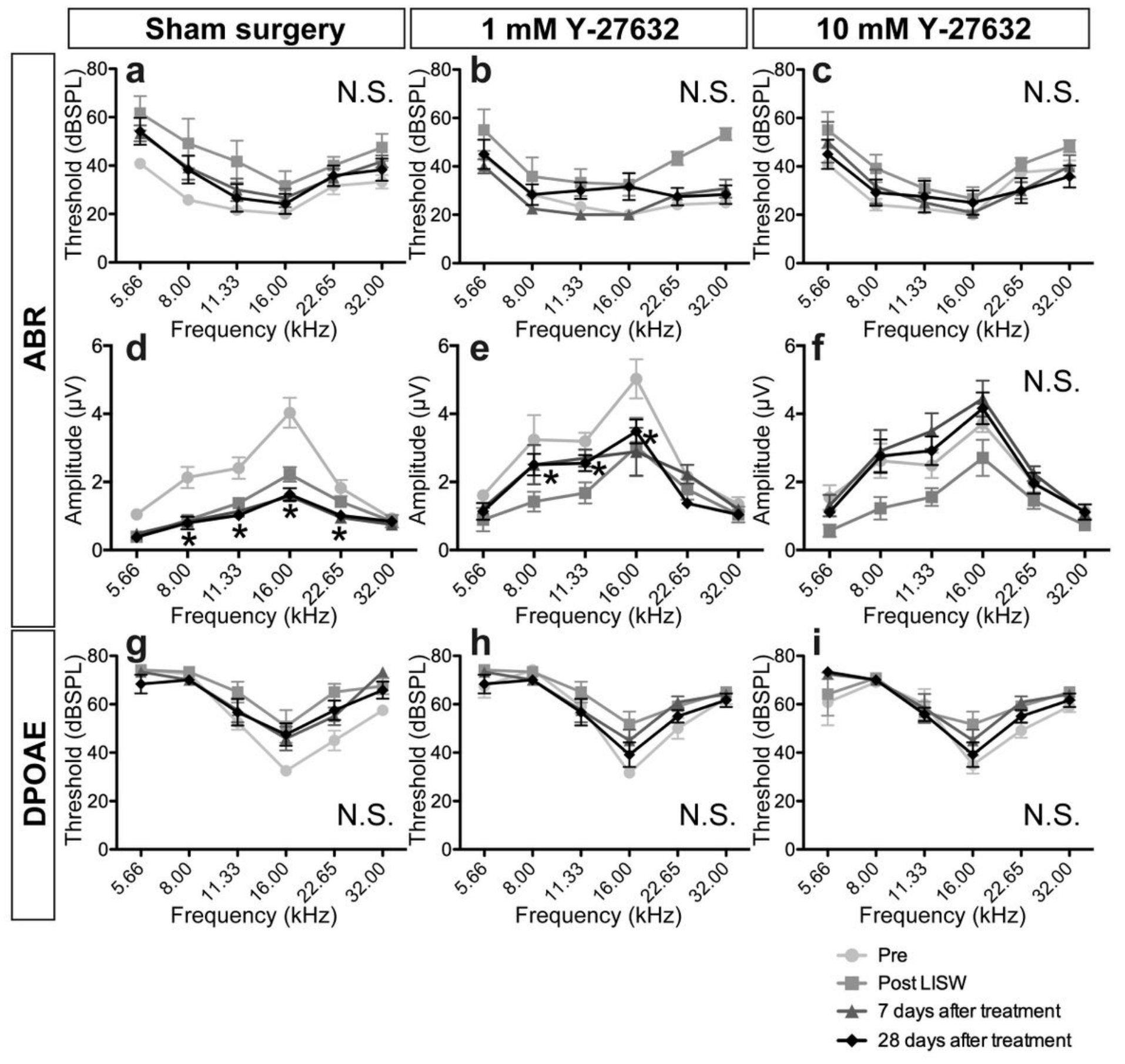

Figure 3

Measurement of hearing function using ABR and DPOAE before and after Y-27632 treatment. (a-c) Changes in ABR threshold at various timepoints shown in Figure 2a. The ABR thresholds at 28 days after Y-27632 treatment (filled diamonds) are not significantly different from those at pre-LISW exposure (filled circle) in any treatment groups, although the temporal threshold shifts at 1 day after LISW exposure can be observed. (d-f) ABR wave I amplitudes at the same timepoints as in a-c. Similar to the ABR threshold changes, a decrease in temporal amplitude can be observed at 1 day after LISW exposure in any groups. However, in the $1 \mathrm{mM} \mathrm{Y-27632-treated} \mathrm{group,} \mathrm{the} \mathrm{amplitude} \mathrm{at} 28$ days after treatment (filled diamonds) 
from $8 \mathrm{kHz}$ to $16 \mathrm{kHz}$ is still significantly (asterisks) lower than that at pre-LISW exposure (filled circle). In $10 \mathrm{mM}$ Y-27632 treatment group, the amplitude at 28 days after treatment (filled diamonds) at any frequencies is not significantly different from that at pre-LISW exposure (filled circle). ( $g-h)$ Changes of DPOAE threshold at various timepoints are shown in Figure 2a. No significant elevations can be observed in the DPOAE thresholds in any treatment groups. * indicates significant changes in the value at 28 days after treatment than at pre-LISW exposure $(p<0.05$, two-way ANOVA, followed by Bonferroni correction for multiple comparisons). Values are represented as mean \pm SEM. 